\title{
EFSUMB Guidelines on Interventional Ultrasound (INVUS), Part IV EUS-guided interventions: General Aspects and EUS-guided Sampling (short version)
}

\author{
EFSUMB Leitlinien interventioneller Ultraschall (INVUS), Teil IV \\ Endosonografisch gestützte Interventionen: allgemeine Aspekte und \\ endosonografisch gestützte Materialgewinnung (Kurzversion)
}

Authors

Affiliations
C. Jenssen ${ }^{1}$, M. Hocke ${ }^{2}$, P. Fusaroli ${ }^{3}$, O. H. Gilja ${ }^{4}$, E. Buscarini ${ }^{5}$, R. F. Havre ${ }^{6}$, A. Ignee $^{7}$, A. Saftoiu ${ }^{8}$, P. Vilmann 9 , E. Burmester ${ }^{10}$, C. P. Nolsøe ${ }^{11}$, D. Nürnberg ${ }^{12}$, M. D’Onofrio ${ }^{13}$, T. Lorentzen ${ }^{11}$, F. Piscaglia ${ }^{14}$, P. S. Sidhu ${ }^{15}$, C. F. Dietrich ${ }^{16,17}$

Affiliation addresses are listed at the end of the article.

\section{Key words}

- guideline

endoscopic ultrasound

- EUS-guided sampling

- EUS-guided fine-needle aspiration

- rapid onsite cytopathological evaluation

- cytopathological reporting

- quality indicators

adverse events

\section{Bibliography}

DOI http://dx.doi.org/

10.1055/s-0035-1553788

Published online: 2016

Ultraschall in Med 2016; 37 :

157-169 ๑ Georg Thieme

Verlag KG Stuttgart · New York .

ISSN 0172-4614

\section{Correspondence}

Prof. Dr. med. Christoph F.

\section{Dietrich}

Med. Klinik 2,

Caritaskrankenhaus Bad

Mergentheim

Uhlandstr. 7

D-97980 Bad Mergentheim

Germany

Tel.: ++ 49/(0)7931/58-22 01/

2200

Fax: ++49/(0)7931/58-22 90

Christoph.dietrich@ckbm.de

\section{Abstract \\ $\nabla$}

The fourth part of the European Federation of Societies for Ultrasound in Medicine and Biology (EFSUMB) Guidelines on Interventional Ultrasound describes general aspects of endoscopic ultrasound-guided diagnostic and therapeutic interventions and assesses the evidence for endoscopic ultrasound-guided sampling. Endoscopic ultrasound combines the most advanced high-resolution ultrasound imaging of lesions within the wall and in the vicinity of the gastrointestinal tract and safe and effective fine needle-based tissue acquisition from these lesions. The guideline addresses the indications, contraindications, techniques, adverse events, training and clinical impact of EUS-guided sampling. Advantages and drawbacks are weighed in comparison with image-guided percutaneous biopsy. Based on the most current evidence, clinical practice recommendations are given for crucial preconditions and steps of EUSguided sampling as well as for safe performance. Additionally, the guideline deals with the principles and reliability of cytopathological reporting in endoscopic ultrasound-guided sampling (short version; the long version is published online).

\section{Introduction: Diagnostic and therapeutic EUS-guided interventions \\ $\nabla$}

This is the first of two guidelines (parts IV and V) within the framework of the European Federation of Societies for Ultrasound in Medicine and Biology (EFSUMB) Guidelines on Interventional Ultrasound describing endoscopic ultrasound (EUS)guided diagnostic and therapeutic interventions.

\section{Zusammenfassung \\ $\nabla$}

Der vierte Teil der Leitlinien der European Federation of Societies for Ultrasound in Medicine and Biology (EFSUMB) zur interventionellen Sonografie beschreibt allgemeine Aspekte endosonografisch gestützter diagnostischer und therapeutischer Interventionen und bewertet die Evidenz für die endosonografisch gestützte Materialgewinnung. Die Endosonografie vereint fortgeschrittene hochauflösende Ultraschallbildgebung von Läsionen in der Wand des Gastrointestinaltrakts und in dessen Umgebung mit der Möglichkeit, daraus mit Feinnadeltechniken effizient und risikoarm Material für die feingewebliche Diagnostik zu entnehmen. Die Leitlinie thematisiert Indikationen, Kontraindikationen, Techniken, unerwünschte Ereignisse, Training und klinischen Stellenwert der endosonografisch gestützten Materialgewinnung. Dabei werden Vor- und Nachteile im Vergleich zur perkutanen bildgebend gestützten Biopsie abgewogen. Auf der Grundlage der aktuellen Evidenz werden für die klinische Praxis Empfehlungen zu den entscheidenden Voraussetzungen und Schritten sowie für eine sichere Durchführung der endosonografisch gestützten Materialgewinnung gegeben. Ergänzend adressiert die Leitlinie Prinzipien und Verlässlichkeit zytopathologischer Befundung von durch endosonografisch gestützte Biopsie gewonnenem Material (Kurzversion; die Langversion ist online publiziert).

Part IV deals with the indications and clinical impact of EUS-guided sampling ${ }^{1}$ and gives evidencebased recommendations for the safe and efficient performance of this technique based on the available evidence at the time of manuscript prepara-

\footnotetext{
1 Term is explained in the addendum on terminology (online version only).
} 
tion. It is complemented by a guideline on EUS-guided therapeutic interventions (part V) [1]. Methods of guideline development are described in the introduction to the EFSUMB Guidelines on Interventional Ultrasound (INVUS) [2]. Levels of evidence (LoE) and Grades of Recommendations (GoR) have been assigned according to the Oxford Centre for Evidence-based Medicine criteria (March 2009 edition) [http://www.cebm.net/oxford-centreevidence-based-medicine-levels-evidence-march-2009].

\section{General considerations}

Endoscopic ultrasound (EUS; synonym: endosonography) ${ }^{2}$ represents an interdisciplinary high-performance imaging method which combines endoscopy and ultrasound by using special transducers mounted at the tip of the endoscope [3 -9]. With the introduction of EUS-guided fine-needle aspiration (EUS-FNA) ${ }^{3}$ in 1992 [10], it became possible to obtain a definite cytopathological or histopathological diagnosis of mediastinal, abdominal, retroperitoneal and pelvic lesions, establishing EUS-FNA as a crucial procedure. Further advances in 1996, allowed EUS-guided celiac plexus neurolysis [11], EUS-guided drainage of GI tract-adjacent fluid collections and pancreatic pseudocysts [12]. A first experience with endobronchial ultrasound-guided transbronchial fine-needle aspiration (EBUS-TBNA) ${ }^{4}$ was reported in 2003 [13].

\section{Equipment and setup}

Linear echoendoscopes have an ultrasound examination field in the longitudinal axis of the echoendoscope, allowing performance of needle-based diagnostic and therapeutic interventions through real-time visualization [14]. A wide variety of accessory equipment for EUS-guided interventions is commercially available [15]. For endobronchial ultrasound (EBUS) specifically designed linear echoendoscopes based on flexible bronchoscopes are available [16]. A therapeutic echoendoscope with a forwardviewing 90-degree curved array at the front of the scope has a potential advantage of allowing interventions with an axial application of force during needle insertion and stenting, with comparable imaging quality and equal performance in EUS-guided drainage of pancreatic pseudocysts being reported [17 - 19].

Both rigid (linear and curved-array) ultrasound probes with a biopsy channel as well as flexible linear and forward-viewing echoendoscopes can be used for transrectal EUS-guided interventions [20 - 27].

\section{Recommendation 1}

Linear echoendoscopes are indispensable for EUS-guided sampling and injection treatments (LoE 5, GoR D). Strong consensus (100\%).

\section{Recommendation 2}

For one-step EUS-guided drainage procedures, a large channel therapeutic linear echoendoscope is recommended (LoE 5, GoR D). Strong consensus (100\%).

\section{Patient preparation and monitoring}

Patient preparation is identical to flexible endoscopy, with fasting for 6 hours, detailed informed consent, management of anticoagulation and antiplatelet therapy, indication for prophylactic antibiotics, and need for sedation [28]. The American Society of Gastrointestinal Endoscopy (ASGE) has defined several evidence-based preprocedural quality indicators [29].

EUS (including EBUS) can be safely performed using conscious sedation (midazolam) perhaps combined with an opioid (fentanyl or pethidine) [30-32]. Alternatively, propofol sedation is a more efficient approach, with better patient and operator satisfaction and a good safety profile, especially for lengthy interventions like therapeutic EUS [31, 33 - 41]. Endoscopist- and nurse-administered propofol sedation has been shown to be safe and effective in EUS and EUS-guided interventions (including EBUS-TBNA) in both average-risk and high-risk patients [36, 37, 42-44]. National legal restrictions in many countries do not permit non-anaesthesiologist administration of propofol $[45,46]$.

\section{Recommendation 3}

Carefully monitored conscious or deep sedation should be routinely offered to all patients undergoing EUS-guided interventions (LoE 1b, GoR B). Strong consensus (100\%).

\section{Recommendation 4}

Controlled propofol sedation should be used with due consideration of national legal regulations, and experience and training of the medical personnel (LoE 5, GoR D). Strong consensus $(100 \%)$.

\section{Platforms for EUS-guided diagnostic and therapeutic} interventions

Needles for EUS-guided sampling are available with a size of 25 19 Gauge $(G)$ [14, 15, 47 - 49]. "Histology needles" include: trucut needles, needles with side fenestration and reverse bevel technology [50 - 57] or with shark mouth-like needle tip geometry. Therapeutic EUS-guided interventions commence with puncture of the targeted lesion for initial access, followed by therapeutic injection or a guidewire-assisted drainage procedure. $19 \mathrm{G}$ needles are normally used, allowing passage of 0.035 inch guidewires.

\section{Indications and contraindications}

EUS has evolved as a diagnostic and therapeutic procedure with substantial clinical impact, altering management in a number of patients [3, 29, 57-63]. Appropriate (evidence-based) indications are listed in $\bullet$ Table 1 [3, 7-9, 29, 57, 64-69].

Absolute contraindications of EUS are similar with those of conventional advanced endoscopy procedures. Specific contraindications of EUS-guided sampling and therapeutic interventions are related to unacceptable risks of bleeding, infection, and perforation ( Table 2; section 5) [8, 9, 14, 66, 70, 71], which should be assessed prior to the intervention on an individual basis weighing procedural risk vs. clinical impact.

\footnotetext{
2 Term is explained in the addendum on terminology (online version only).

3 Term is explained in the addendum on terminology (online version only).

${ }^{4}$ Term is explained in the addendum on terminology (online version only)
} 
Table 1 Established indications of endoscopic ultrasound.

\begin{tabular}{|c|c|}
\hline \multicolumn{2}{|c|}{ diagnostic evaluation (including EUS-guided sampling) } \\
\hline \multirow{5}{*}{$\begin{array}{l}\text { pancreatico-biliary } \\
\text { disorders }\end{array}$} & obstructive jaundice/biliary stricture \\
\hline & suspected bile duct stones \\
\hline & biliary and idiopathic acute pancreatitis \\
\hline & (early) chronic pancreatitis \\
\hline & solid and cystic focal pancreatic lesions \\
\hline \multirow{2}{*}{$\begin{array}{l}\text { oesophagogastro- } \\
\text { duodenal tumours }\end{array}$} & subepithelial tumours \\
\hline & enlarged gastric folds/wall thickening \\
\hline \multirow[t]{2}{*}{ mediastinal diseases } & primary central lung/mediastinal tumours \\
\hline & mediastinal lymphadenopathy \\
\hline rectal diseases & $\begin{array}{l}\text { fistulas, abscesses, extraluminal tumours, } \\
\text { subepithelial tumours }\end{array}$ \\
\hline \multicolumn{2}{|c|}{ staging of various cancers } \\
\hline $\begin{array}{l}\text { upper gastrointesti- } \\
\text { nal cancer }\end{array}$ & $\begin{array}{l}\mathrm{T} \text { (tumour) and } \mathrm{N} \text { (nodal) stage, } \mathrm{M} \text { (metastatic) } \\
\text { stage (celiac trunk lymph nodes, left adrenal, } \\
\text { left liver lobe, ascites, etc.) }\end{array}$ \\
\hline lung cancer & $\begin{array}{l}\text { T and N stage (EUS and EBUS), M stage } \\
\text { (abdominal lymph nodes, left adrenal, left } \\
\text { liver lobe) }\end{array}$ \\
\hline rectal cancer & T and $\mathrm{N}$ stage \\
\hline $\begin{array}{l}\text { pancreatico-biliary } \\
\text { cancers }\end{array}$ & $\begin{array}{l}\text { T and } \mathrm{N} \text { stage, } \mathrm{M} \text { stage (left liver lobe, ascites, } \\
\text { mediastinal lymph nodes, etc.); evaluation of } \\
\text { resectability and vascular invasion }\end{array}$ \\
\hline \multicolumn{2}{|c|}{ EUS-guided therapeutic interventions } \\
\hline \multirow[t]{3}{*}{ drainage procedures } & $\begin{array}{l}\text { transmural drainage of (per-)pancreatic and } \\
\text { peri-intestinal fluid collections (pseudocysts, } \\
\text { walled-off pancreatic necroses, abscesses, } \\
\text { postoperative fluid collections) }\end{array}$ \\
\hline & bile duct and gallbladder drainage \\
\hline & pancreatic duct drainage \\
\hline \multirow[t]{3}{*}{ injection treatment } & celiac plexus/ganglia neurolysis and block \\
\hline & $\begin{array}{l}\text { glue or coil embolization of bleeding varices, } \\
\text { visceral pseudoaneurysms, and other vascular } \\
\text { lesions }\end{array}$ \\
\hline & $\begin{array}{l}\text { ethanol ablation of small pancreatic } \\
\text { insulinomas }\end{array}$ \\
\hline other interventions & placement of fiducials \\
\hline
\end{tabular}

Table 2 Indications and contraindications of EUS-guided sampling.

\begin{tabular}{|lll|}
$\begin{array}{l}\text { frequent/ } \\
\text { established } \\
\text { indications }\end{array}$ & $\begin{array}{l}\text { less frequent/ emerging } \\
\text { indications }\end{array}$ & contraindications \\
\hline $\begin{array}{l}\text { pancreatic } \\
\text { solid lesions }\end{array}$ & retroperitoneal masses & absolute \\
\hline $\begin{array}{l}\text { pancreatic } \\
\text { cystic lesions }\end{array}$ & mediastinal masses & $\begin{array}{l}\text { no impact on patient } \\
\text { management }\end{array}$ \\
\hline $\begin{array}{l}\text { lymph nodes } \\
\text { lack of informed } \\
\text { consent }\end{array}$ \\
\hline $\begin{array}{l}\text { subepithelial } \\
\text { tumours }\end{array}$ & perianastomotic masses & $\begin{array}{l}\text { adrenal gland masses } \\
\text { (risk of infection) }\end{array}$ \\
\hline & $\begin{array}{l}\text { liver masses } \\
\text { nodules and peritoneal }\end{array}$ & $\begin{array}{l}\text { relative } \\
\text { severe coagulopathy }\end{array}$ \\
\hline & $\begin{array}{l}\text { gastrointestinal wall } \\
\text { thickening }\end{array}$ & $\begin{array}{l}\text { continued oral } \\
\text { anticoagulation }\end{array}$ \\
\hline & $\begin{array}{l}\text { bile duct strictures/ } \\
\text { lesions }\end{array}$ & $\begin{array}{l}\text { continued treatment } \\
\text { with ADP antagonists } \\
\text { (clopidogrel, prasu- } \\
\text { grel, ticagrelor) }\end{array}$ \\
\hline & kidney masses & $\begin{array}{l}\text { large vessels in the ex- } \\
\text { pected needle track }\end{array}$ \\
\hline & focal splenic lesions & \\
\hline
\end{tabular}

\section{Recommendation 5}

As EUS is an advanced invasive procedure, it requires a proper indication, assessment of individual risks and contraindications, detailed informed consent, considerate management of antithrombotic therapy and antibiotic prophylaxis, and a preinterventional team time-out ${ }^{5}$ (LoE 5, GoR D). Strong consensus $(100 \%)$

\section{Education and training in endoscopic ultrasound Preconditions for performing EUS}

EUS has evolved into an advanced endoscopic procedure requiring structured training as supported by the ASGE core curriculum for EUS [72]. Certain conditions need to be attained prior to commencing an EUS program; state-of-the-art EUS equipment and accessories should be in place, previous EUS experience and training (>12 months) with proficiency in basic EUS should be available, the presence of a multidisciplinary team with expertise in gastroenterology, pulmonary medicine, oncology, surgery, imaging and pathology is desired.

\section{Methods and models for training}

Various approaches for structured training have been described. Support includes EUS textbooks and atlases, DVDs and online resources [7 - 9, 73 - 78]. Computer-based simulator training, EUSguided sampling and therapeutic intervention training with animal models and supervised hands-on may facilitate improved learning for clinical application and may minimize false-negative sampling in patients [73, 76, 79-93]. Objective assessment tools with rating scales for various steps of EUS-guided sampling have been developed to measure competency for mediastinal staging of NSCLC $[83,85,90,91,94-96]$.

\section{Learning curve and minimum number of supervised procedures}

Reports on the skill acquisition of individuals for EUS [97, 98] and for EUS-FNA of solid pancreatic lesions $[99,100]$ challenge the ASGE recommendations that comprehensive competence in all aspects of EUS requires at least 150 supervised cases, including 50 EUS-FNA [101]. Also for EBUS-TBNA, studies show significant variation in learning curves [96] with an acceptable $90 \%$ accuracy achieved after performing $50-80$ procedures [102 - 105].

\section{Recommendation 6}

Education and training for EUS procedures should be performed systematically in a standardized fashion. Various learning methods should be used, including textbooks and on-line resources, and EUS phantoms, before performing hands-on human cases (LoE 5, GoR D). Strong consensus (100\%).

\section{Recommendation 7}

Standardized criteria and assessment tools should be developed and applied for assessing skills of individual trainees before credentialing competency (LoE 1b, GoR B). Strong consensus $(100 \%)$

\footnotetext{
${ }^{5}$ Term is explained in the addendum on terminology (online version only).
} 


\section{Recommendation 8}

At least 50 EUS-guided sampling procedures are needed in order to attain basic competency in EUS-guided interventions (LoE 5, GoR D). Strong consensus (100\%).

\section{Quality indicators and benchmarking}

Useful quality indicators for EUS-guided sampling may be:

a) percentage of appropriate indications for performing EUSguided sampling due to guidelines ( $\boldsymbol{0}$ Table 2) [29]

b) prospective documentation of adverse events after EUS-guided sampling (performance target: > 98\%) [29]

c) incidence of adverse events after EUS-guided sampling (performance targets: acute pancreatitis $<2 \%$, clinically significant bleeding < $1 \%)[29,48,66]$

d) frequency of algorithmic EUS-guided sampling of both suspected metastatic disease and the primary tumour in cases, in which results of EUS staging would impact further clinical management (performance target: > 98\%) [29]

e) the yield of malignant diagnoses ${ }^{6}$ in EUS-guided sampling of solid pancreatic masses and the sensitivity for diagnosis of pancreatic cancer (performance targets: $\geq 70$ and $85 \%$, respectively) $[29,48,57,66,106]$

f) the percentage of adequate samples ${ }^{7}$ (performance target: $\geq 85 \%$ ) $[29,48,66]$ and

g) the frequency of inconclusive cytopathological diagnoses ${ }^{8}$ (atypical, suspicious: $\leq 10 \%$ ) [48, 66, 107].

Particular quality parameters for EUS-guided therapeutic interventions have not as yet been established, but should be derived from those for EUS-guided sampling and advanced endoscopic and interventional procedures $[29,108]$.

\section{Recommendation 9}

Appropriate indicators should be implemented to monitor the quality of EUS-guided interventions (LoE 5, GoR D). Strong consensus (100\%).

\section{EUS-guided sampling: Indications and clinical impact} $\nabla$

\section{General indications of EUS-guided sampling}

EUS has high accuracy for the diagnosis and staging of benign and malignant conditions within and outside the gastrointestinal tract ( $\bullet$ Table 2) [61, 62]. EUS-guided sampling relies on its ability to obtain specimens with either fine-needle aspiration (FNA) or fine-needle biopsy $(\mathrm{FNB})^{9}[57,66]$.

Several prospective and retrospective studies and meta-analyses have substantiated the key importance of EUS-guided sampling particularly in suspicious nodal disease for staging, guiding treatment, and predicting outcome in non-small cell lung cancer (NSCLC) [109-114], various extrathoracic malignancies [115, 116], upper gastrointestinal cancer [117, 118], rectal cancer [23], as well as proving recurrence of malignancy $[20,119-122]$. Speci-

\footnotetext{
${ }^{6}$ Term is explained in the addendum on terminology (online version only).

${ }^{7}$ Term is explained in the addendum on terminology (online version only).

${ }^{8}$ Term is explained in the addendum on terminology (online version only).

${ }^{9}$ Term is explained in the addendum on terminology (online version only).
}

men provided by EUS can be examined by immunohistochemical and biological marker analysis to identify specific tumour characteristics, facilitating personalized treatment [123-131].

\section{Recommendation 10}

EUS-guided sampling should be considered for tissue diagnosis of lesions in or adjacent to the gastrointestinal tract when the result is likely to alter clinical management (LoE 2a, GoR B). Strong consensus (100\%).

\section{EUS-guided sampling for primary diagnosis}

Pancreatic solid lesions

Solid pancreatic lesions suspected as ductal pancreatic adenocarcinoma (PDAC) are typically targets for EUS-guided sampling. Metastases and/or primary lesion sampling is indicated when the lesion is non-resectable allowing appropriate radiotherapy/chemotherapy. EUS-guided sampling of potentially resectable tumours will follow local protocols to obtain confirmation of malignancy prior to surgical intervention.

EUS has a high positive predictive value (PPV) and a fair negative predictive value (NPV) in diagnosing PDAC. Several studies report a sensitivity between $85-93 \%$ and a specificity between $96-$ $100 \%[132-136]$.

EUS-guided sampling is a reliable method to differentiate PDAC from neuroendocrine pancreatic tumours and other rare pancreatic neoplasms (non-PDAC), each with differing outcomes and alternative treatment strategies [137 - 140].

\section{Recommendation 11}

In pancreatic masses, EUS-guided sampling should be preferred in potentially resectable lesions and in unresectable lesions in which percutaneous biopsy is not feasible (LoE $2 b$, GoR C). Strong consensus (100\%).

\section{Recommendation 12}

EUS-guided sampling should be preferred over percutaneous biopsy in pancreatic masses prior to neoadjuvant radiotherapy/chemotherapy (LoE 2b, GoR C). Strong consensus (100\%).

\section{Recommendation 13}

EUS-guided sampling is recommended in potentially resectable, pancreatic masses that are atypical for pancreatic ductal adenocarcinoma (LoE 3b, GoR C). Strong consensus (100\%).

\section{Pancreatic cystic lesions}

There is a wide differential diagnosis of pancreatic cystic lesions (PCL) including both benign and malignant [141 - 143].

EUS-guided sampling aims to differentiate mucinous from nonmucinous and malignant from benign lesions. For best diagnostic accuracy, EUS-FNA cytology and biochemical aspirate assays should be reviewed in combination with the EUS findings, clinical history and other imaging techniques [144 - 146].

Levels of carcinoembryonic antigen (CEA) and amylase yield a suboptimal diagnostic accuracy of $60-86 \%$ for distinguishing between mucinous and non-mucinous PCL $[147-150]$. The spe- 
cificity of cytological diagnosis of malignant PCL is adequate, but the sensitivity is low ( $88-97 \%$ and $51-65 \%$, respectively) [150 - 153]. The combination of molecular analysis and CEA or cytology has a better performance for the diagnosis of neoplastic mucinous PCL than either of the individual tests [154-156].

\section{Recommendation 14}

Biochemical, cytological, and molecular analysis of EUS aspirates from pancreatic cystic lesions may facilitate differentiation between mucinous and non-mucinous cysts and evaluation of the malignancy risk (LoE 2a, GoR B). Strong consensus (100\%).

\section{Biliary cancer}

Several studies report successful EUS-guided sampling of gallbladder wall lesions [157 - 161], extrahepatic cholangiocarcinoma and indeterminate biliary strictures [157, 162 -173], as well as tumours of the papilla of Vater [174], but with wide variation in sensitivity (43-100\%) [175]. EUS-guided sampling proved to be successful following failed or negative ERCP-guided brushing or biopsy [163, 165 - 169, 175]. Comparison of EUS-guided sampling with that of ERCP-guided techniques revealed a better outcome for EUS in gallbladder cancer, biliary strictures produced by pancreatic cancer rather than cholangiocarcinoma, and in EUS-visible masses, with a combined approached considered clinically appropriate [159, 161, 164, 173, 175]. EUS-guided sampling of hilar lymph nodes may be used to diagnose and stage suspected biliary cancer $[159,160,162,168,172,176]$.

\section{Recommendation 15}

Complementary to ERCP-guided brushing and biopsy, EUSguided sampling may be used for diagnosis of indeterminate biliary strictures and gallbladder masses (LoE 2a, GoR B). Strong consensus (100\%).

\section{Subepithelial gastrointestinal tumours}

Differential diagnosis of gastrointestinal subepithelial tumours (SET) includes leiomyoma, schwannoma, gastrointestinal stromal tumour (GIST), lipoma, ectopic pancreas, neuroendocrine tumour, cyst and others [177 - 179]. Whilst a reliable differential diagnosis is possible on EUS features alone in some SETs, so, with the frequently occurring hypoechoic SET originating from the $4^{\text {th }}$ layer (muscularis propria), i.e. GIST, leiomyoma and schwannoma, diagnosis may be problematic [177-179]. EUS-guided sampling combines smear cytology (SC) with immunohistochemistry for maximal diagnostic accuracy. The diagnostic yield remains suboptimal using standard EUS needles of various diameters, ranging from 20 [180] to $93 \%$ [181], as retrieved tissue is not always sufficient to allow immunohistochemical analysis [181 - 195]. The European Society of Gastrointestinal Endoscopy (ESGE) guideline on EUS-guided sampling in gastroenterology states that in hypoechoic SET of the stomach $<20 \mathrm{~mm}$ the usefulness of EUS-guided sampling is limited due to the moderate diagnostic yield and lacking capability to determine the mitotic index [59].

\section{Recommendation 16}

EUS-guided sampling may be used for differential diagnosis of subepithelial gastrointestinal tumours $\geq 20 \mathrm{~mm}$ in cases with high surgical risk or suspected non-resectability (LoE 2b, GoR C). Strong consensus (100\%).

\section{Lung cancer}

In suspected lung cancer conventional techniques (bronchoscopic or percutaneous-transthoracic biopsy) fail to establish a histological diagnosis in up to $1 / 3$ of patients. EUS allows effective transoesophageal or transbronchial guidance for sampling of centrally located lung masses or lymph nodes adjacent to the oesophagus or the respiratory tract [196-203]. Subtyping of non-small cell lung cancer is the basis of personalized oncological treatment and is feasible using EUS-guided sampling in 77 90.6\% [204-206]. Genotyping was possible using EUS-/EBUS fine-needle aspirates in $77-98.4 \%$ [124, $204-209]$.

\section{Recommendation 17}

In suspected lung cancer EUS-guided sampling (EUS-FNA, EBUS-TBNA) of centrally-located mass lesions or suspicious lymph nodes is recommended to establish a definite tissue diagnosis complementing other diagnostic techniques (LoE 2b, GoR B). Strong consensus (100\%).

\section{Mediastinal and abdominal lymphadenopathy of un-} known origin

For lymph node classification as malignant or benign, EUS-FNA has a higher accuracy than EUS features alone [210, 211]. A meta-analysis ( $n=9310)$ showed significant improvement in evaluating mediastinal lymphadenopathy with EUS-guided sampling over EUS features of lymph nodes (sensitivity 88 vs. $84.7 \%$; specificity 96.4 vs. $84.6 \%$ ) [212]. Several studies and meta-analyses have demonstrated a high diagnostic yield and accuracy of EUS-guided sampling (EUS-FNA and EBUS-TBNA) for the evaluation of indeterminate mediastinal and abdominal lymphadenopathy, with or without a malignant disease background [114, $116,120,212-234]$.

\section{Recommendation 18}

EUS-guided sampling (EUS-FNA, EBUS-TBNA) is recommended as the primary diagnostic technique for tissue diagnosis of mediastinal or abdominal lymphadenopathy of unknown aetiology (LoE 3a, GoR B). Strong consensus (100\%).

\section{EUS-guided sampling for tumour staging}

Algorithmic approach

For tumour staging an algorithmic approach of EUS-guided sampling following the TNM classification of the respective malignant tumour is sensible. Potential sites of metastases within reach of EUS-FNA should be examined and sampled first, followed by regional lymph node stations, in which demonstration of metastatic involvement would alter management [76, 235 237]. This systematic approach in an inverse TNM order meets concerns that needle contamination with tumour cells from the first sampling target, from the instrumental channel or from gas- 
trointestinal fluid could contribute to false-positive results from the subsequent targeted lesions [238-241].

\section{Mediastinal lymph nodes}

In the absence of distant metastases, mediastinal lymph node involvement is the most important factor affecting the management and outcome of patients with NSCLC [236]. Oesophageal EUS has a high sensitivity and specificity for lymph node involvement when found in the posterior mediastinum, aortopulmonary, subcarinal, and periesophageal regions [68, 212, 236, 242 244]. Meta-analyses report a sensitivity and specificity between $83-89 \%$ and $96.4-100 \%$, respectively, for nodal staging of lung cancer by EUS-guided sampling [110-114, 225, 236, 245]. EUS has been shown to be more accurate than $\mathrm{CT}$ in defining the disease stage [244, 246, 247]. EUS-guided sampling impacts treatment choice and survival in patients with NSCLC [247 - 250]. Oesophageal EUS and EBUS in combination allow targeting of nearly all relevant mediastinal lymph node locations [251 - 254]. Several studies and meta-analyses have shown that this combined approach improves lymph node staging, compared with either technique when used alone [112, 114, 236, 237, 250, 255 - 263]. There is a $21 \%$ sensitivity increase for mediastinal nodal staging in proven or suspected lung cancer by performing combined endobronchial and oesophageal endosonography-guided sampling compared with the oesophageal approach alone, and a $13 \%$ increase compared with EBUS-TBNA alone [237].

Both oesophageal EUS and EBUS have also been successfully used for the assessment of tumour spread to mediastinal lymph nodes (M1 disease) in a variety of extra-thoracic malignant disease $[115,213,220,223,264-268]$. A meta-analysis ( $n=533$ patients) showed a sensitivity of $85 \%$ and a specificity of $99 \%$ of EBUSTBNA for the diagnosis of mediastinal lymph node metastases of extra-thoracic malignancies [116].

EUS-FNA of mediastinal lymph nodes for the N staging of oesophageal cancer is more sensitive and accurate than EUS alone and significantly impacts therapeutic decisions [269-271].

\section{Abdominal lymph nodes}

Previous studies have shown high accuracy and clinical impact of EUS-guided sampling of celiac lymph nodes in oesophageal cancer staging [272-274]. According to the current TNM classification, these are included in the $N$ category [275], with EUS-guided sampling only being useful in selected cases.

In addition to EUS-guided sampling of mediastinal lymph nodes, sampling of abdominal lymph nodes in gastric cancer may alter staging classification. Malignant involvement of distant lymph nodes is indicative of metastatic disease and resigns the patient to palliative care $[117,118]$.

\section{Adrenal glands and liver masses}

EUS-guided sampling of adrenal masses can provide valuable information for the staging of lung cancer and other malignancies. Particularly in lung cancer, 30 - $80 \%$ of adrenal masses are adenomas [276]. Sampling from the left adrenal gland is viable and safe; sampling from the right adrenal gland has also been reported. The diagnostic yield of EUS-guided sampling ranges from $76-100 \%$. Further management is altered in approximately $50 \%$ of lung cancer patients [277 - 289].

EUS and EUS-guided sampling are superior to CT in detecting small liver metastases $[290,291]$. The diagnostic yield is reported between $80-98 \%$ with a substantial effect on clinical management [292-298].
Other metastatic locations and diagnosis of recurrent malignant disease

EUS and EUS-guided sampling may play a pivotal role in the detection and diagnosis of disease recurrence or late distant spread of gastrointestinal cancer [299]. A high accuracy with clinical impact of EUS-guided sampling has been shown in establishing recurrent malignancy following curative treatment [20, 119-122, $300-304]$, and metastatic disease, in particular affecting the pancreas [139, 303, $305-307]$, spleen [308, 309], pelvis [24, 26, $310]$ and peritoneal space [297, $311-322]$.

\section{Recommendation 19}

Combined EUS-guided sampling (EBUS-TBNA and EUS-FNA) is recommended for complete nodal staging of non-small cell lung cancer (LoE 1a, GoR A). Strong consensus (100\%).

\section{Recommendation 20}

EUS-guided sampling (EBUS-TBNA and EUS-FNA) may be used in mediastinal nodal and metastatic staging of other malignancies (LoE 3a, GoR B). Strong consensus (100\%).

\section{Recommendation 21}

EUS-guided sampling may be used for the detection of recurrence of malignancies ( $\mathrm{LoE} 3 \mathrm{~b}, \mathrm{GoR} \mathrm{C}$ ). Strong consensus (100\%).

\section{Recommendation 22}

When biopsy of suspected metastatic lesions for staging purposes is contemplated, penetration of the primary tumour should be avoided (LoE 5, GoR D). Strong consensus (100\%).

\section{Recommendation 23}

In cases with suspected distant and/or nodal metastases, algorithmic sampling should be performed beginning with the potentially most advanced metastatic site and following an inverse TNM order (LoE 5, GoR D). Strong consensus (100\%).

\section{EUS-guided sampling: Needle choice, sampling tech- niques and other factors influencing diagnostic yield $\nabla$}

Choice of needle type and diameter

There are four types of needles commercially available for EUSguided sampling: standard aspiration needles (19 G, 22 G, 25 G for EUS-FNA; $21 \mathrm{G}$ and $22 \mathrm{G}$ for EBUS-TBNA), aspiration needles with a core trap and reverse bevel technology (19 G, 20 G, 22 G, $25 \mathrm{G})$, aspiration needles with a shark mouth needle tip geometry (19 G, 22 G, 25 G), and trucut needles (19 G).

\section{EUS-guided fine-needle aspiration for cytopathological processing}

Standard aspiration needles were originally designed to obtain cellular material for cytopathological examinations [66, 323, 324]. Cytological yield and diagnostic accuracy, in studies using predominantly $22 \mathrm{G}$ aspiration needles, are reported to be high 
for solid pancreatic lesions and metastatic lymph nodes, satisfactory for biliary mass lesions, but only moderate for gastrointestinal SET and PCL $[59,66,325,326]$. Currently, the $22 \mathrm{G}$ aspiration needle is most widely used, with increased utilization of $25 \mathrm{G}$ aspiration needles [327 - 329].

$22 \mathrm{G}$ vs. $25 \mathrm{G}$ aspiration needles

Meta-analyses demonstrate slight superiority of the $25 \mathrm{G}$ over the $22 \mathrm{G}$ aspiration needle for EUS-FNA of solid pancreatic lesions $[330,331]$.

\section{G vs. 22 G and $25 \mathrm{G}$ aspiration needles}

There was no difference in diagnostic accuracy between the $19 \mathrm{G}$ and the $22 \mathrm{G}$ aspiration needle by intention-to-treat analysis, but the $19 \mathrm{G}$ was superior to the $22 \mathrm{G}$ needle in terms of the amount of cellular material and diagnostic accuracy by per-protocol analysis (following exclusion of 5 technical failures, all with pancreatic head lesions). Further analysis favoured the $19 \mathrm{G}$ aspiration needle for the sampling of lesions within the pancreatic body and tail [332]. A flexible $19 \mathrm{G}$ aspiration needle has been shown to be effective for procuring aspirates from pancreatic head lesions even by the transduodenal route [333 - 335].

$21 \mathrm{G}$ vs. $22 \mathrm{G}$ aspiration needles for EBUS-TBNA

A meta-analysis of 5 studies ( $n=1720$ patients) did not reveal significant differences in the diagnostic yield, sample adequacy, or the mean number of needle passes between the 21 and $22 \mathrm{G}$ needles during EBUS-TBNA [336].

\section{EUS-guided tissue acquisition for histopathological pro- cessing}

With increasing requirements of histopathological, immunohistochemical and molecular biological diagnosis [337, 338], three types of "histology needles" have been developed to facilitate acquisition of core cylinders suitable for histopathological examination: trucut needles [50, 51, 339, 340], aspiration needles with a side fenestration (core trap) and reverse bevel technology $[53,55,341,342]$ and aspiration needles with a shark mouth design. Acquisition of tissue suitable for histopathological processing is also possible with standard aspiration needles, not just with "histology needles" [54, 59, 66, 323, 324, 343, 344]. The diagnostic yield for histological material is dependent on the needle diameter.

\section{Recommendation 24}

In a referral EUS centre it is advisable to have needles of different size available, and the choice among different needles should be made considering the anatomical location and the type of the target lesion and the preferred mode of processing of the material (LoE 2b, GoR C). Strong consensus (100\%).

\section{Recommendation 25}

$22 \mathrm{G}$ aspiration needles have a high diagnostic yield, low risk of adverse events, and good technical performance, and therefore should be regarded as the current standard for EUS-guided sampling (LoE 2a, GoR B). Strong consensus (100\%).

\section{Recommendation 26}

$25 \mathrm{G}$ aspiration needles are not inferior to $22 \mathrm{G}$ aspiration needles with respect to cytological yield (LoE $1 \mathrm{a}, \mathrm{GoR} A$ ). The use of $25 \mathrm{G}$ needles should be considered in particular for EUSguided transduodenal sampling of pancreatic head lesions (LoE 2a, GoR C). Strong consensus (100\%).

\section{Recommendation 27}

Due to a high rate of technical failure, the use of nonflexible 19 $\mathrm{G}$ needles is not recommended for transduodenal sampling of pancreatic head masses (LoE 2b, GoR B). Strong consensus $(100 \%)$.

\section{Factors influencing the yield of EUS-guided sampling}

Features of the target lesion

The hypocellular character of the PCL aspirate renders the diagnostic yield and accuracy of EUS-guided sampling of PCL considerably lower than in the case of solid pancreatic lesions.

For gastrointestinal SET the diagnostic yield of EUS-guided sampling (specific diagnosis) is approximately 34\% [187] to $82 \%$ [184], with most studies reporting a yield of $52-63 \%$ [179]. Data on the influence of the size and site of solid pancreatic lesions on EUS-FNA accuracy are conflicting [138, 345 - 348]. The diagnostic accuracy of EUS-FNA for pancreatic cancer is considerably hampered in patients with chronic pancreatitis [349-353]. There are inconsistent results in studies evaluating the influence of biliary stents on EUS-FNA performance characteristics [354, 355].

\section{Sampling technique}

Fanning technique

Sampling of all parts of solid lesions and lymph nodes ("fanning technique") is recommended by the ESGE guidelines on EUSguided sampling in gastroenterology [54]. According to the results of an RCT, significantly fewer passes are required to establish a diagnosis using this particular technique [356].

\section{Recommendation 28}

Fanning technique should be applied in EUS-guided sampling to increase sample adequacy (LoE 2b, GoR C). Strong consensus $(100 \%)$.

Suction

Traditionally negative suction pressure using a $5 \mathrm{ml}$ or $10 \mathrm{ml}$ syringe is used to facilitate EUS-guided tissue acquisition. Alternatively, the fine-needle capillary sampling technique [357] or slow removal of the stylet ("slow-pull technique") [358] may be used to draw cells up into the needle lumen. Applying suction to the needle may increase the cellular yield of the aspirate but, at the same time, potentially increases artefacts and contamination by blood. Applying high negative pressure suction has been suggested to facilitate procurement of core tissue in EUS-FNA and EBUS-TBNA [184, 359 - 363].

An RCT demonstrated that EUS-guided lymph node sampling with suction compared with the fine-needle capillary technique did not improve the likelihood of correct diagnosis, but, due to excessive blood contamination, provided smears of significantly poorer quality [364]. 
For sampling of pancreatic masses using 22 and $25 \mathrm{G}$ aspiration needles, one RCT favoured EUS-guided fine-needle sampling with $10 \mathrm{ml}$ suction over no suction [365]. In a multicentre RCT the adequacy and accuracy of 22 G EUS-FNA from solid pancreatic lesions were significantly better with $20 \mathrm{ml}$ suction compared with $10 \mathrm{ml}$ suction and no suction [366].

\section{Recommendation 29}

For aspiration of cysts and fluid collections, negative pressure suction is recommended (LOE 5, GoR D). Strong consensus $(100 \%)$.

\section{Recommendation 30}

For sampling of solid pancreatic lesions, negative pressure suction should be considered (LOE 1b, GoR A). Strong consensus (100\%).

\section{Recommendation 31}

In lymph nodes and other highly vascularized solid lesions, sampling without applying suction should be considered (LoE 2b, GoR C). Strong consensus (100\%).

\section{Use of a stylet}

No advantage of using a stylet in terms of specimen quality and diagnostic yield has been demonstrated [367-371]. An RCT suggested a lower proportion of adequate samples and a higher probability for blood contamination in needle passes using a stylet compared with needle passes performed without a stylet [367]. A multicentre RCT reported non-inferiority of performing $22 \mathrm{G}$ EUS-FNA of various solid lesions without a stylet compared with using a stylet with respect to the acquisition rate of histological specimens [372].

\section{Recommendation 32}

Aspiration needles may be used with or without a stylet with the same diagnostic yield (LoE 1b; GoR A). Strong consensus (100\%).

\section{Number of needle passes}

The number of passes is determined based on the results of on-site cytopathological evaluation, should this be available (ROSE) ${ }^{10}[54$, $373,374]$. As an alternative to ROSE-guided sampling, a high number $(\geq 7)$ of needle passes is thought to be a predictor of a high diagnostic sensitivity of EUS-FNA [327].

Pancreatic lesions

ROSE study results indicate that diagnosis of a solid pancreatic lesion using standard aspiration needles may require $5-7$ passes [375 - 377]. When using a $25 \mathrm{G}$ aspiration needle for sampling of a solid pancreatic lesion, 2 - 4 needle passes were adequate for a good diagnostic accuracy [378-381]. Using a standard 22 G as-

\footnotetext{
${ }^{10}$ The terms "number of passes" and ROSE are explained in the addendum on terminology (online version only).
}

piration needle, 1 - 2 needle passes were sufficient to achieve a high diagnostic yield and accuracy in $92 \%$ of cases with solid pancreatic lesions [382].

In the case of PCL with no solid component, a single pass and complete cyst fluid aspiration should be performed to prevent cyst infection [54]. In the case of PCL with a solid component, the diagnostic yield is significantly higher for $\geq 2$ needle passes (78\%) compared to one needle pass (44\%) [383].

\section{Non-pancreatic lesions}

In the case of lymph nodes, liver metastases and adrenal lesions, a lesser number of needle passes is sufficient to provide a high diagnostic yield. Approximately 3 needle passes were sufficient for EUS-FNA and EBUS-TBNA of lymph nodes [364, 375, 377, 379, 384].

\section{Recommendation 33}

If ROSE is not available, the number of needle passes should be based on gross visual inspection of the obtained material and the type of target lesion (LoE 2b, GoR C). Strong consensus (100\%).

\section{Targeted sampling}

EFSUMB guidelines recommend the use of contrast-enhanced endoscopic ultrasound (CE-EUS) and of EUS-elastography to facilitate differential diagnosis of solid pancreatic lesions, PCL and lymph nodes [385, 386].

\section{Lymph nodes}

EUS-elastography, currently strain elastography, has been shown to increase the discriminatory ability of normal EUS criteria, and to improve the specificity of lymph node staging in gastrointestinal malignancy $[386,387]$. A meta-analysis calculated a sensitivity of $88 \%$ and specificity of $85 \%$ with EUS-elastography for differentiating between benign and malignant lymph nodes [388]. EFSUMB guidelines suggest using EUS-elastography for identifying suspicious lymph node and/or harder lymph node regions as targets of EUS-guided sampling [386].

\section{Solid pancreatic lesions}

The selection of patients for EUS-guided sampling with a high suspicion of diagnoses other than PDAC is of pivotal importance to avoid unnecessary pancreatic head resection with inherent morbidity and mortality [343].

Contrast-enhanced ultrasound (CEUS) and CE-EUS are able to discriminate hypovascular PDAC from other iso- and hypervascular solid pancreatic lesions (non-PDAC) with an accuracy $>85 \%$ $[385,389]$. A meta-analysis reported the sensitivity and specificity of CE-EUS to diagnose PDAC at 94 and 89\%, respectively [390]. When there is a high suspicion of non-PDAC, patients with solid pancreatic mass lesions which are hypervascular or isovascular in comparison with the surrounding pancreatic parenchyma should be referred to pre-therapeutic EUS-guided sampling. Negative results of EUS-guided sampling of solid pancreatic lesions which are hypovascular on CE-EUS should be considered falsenegative, initiating repeat sampling or proceeding directly to surgery [391, 392]. An RCT demonstrated that CE-EUS-guided FNA of solid pancreatic lesions is more efficient than conventional EUS-FNA with fewer needle passes required to obtain a diagnostic sample [393]. 
EUS-elastography has an high sensitivity, but only a moderate specificity for differentiating malignant from benign solid pancreatic lesions [387]. Meta-analyses report a sensitivity of 95 $97 \%$ and a specificity of $67-76 \%$ [394 - 398]. EFSUMB guidelines recommend that in the case of persisting strong clinical suspicion of pancreatic cancer with inconclusive or negative EUS-guided sampling, a hard focal lesion on elastography and/or a hypovascular lesion on CEUS and or CE-EUS indicates the need for repeat EUS-FNA or referral to surgery [386].

\section{Cystic pancreatic lesions}

The presence of epithelial mural nodules, thick walls, and septa has been shown to be highly predictive for malignancy in BDIPMN [399, 400]. International and European guidelines recommend surgical treatment of BD-IPMN in particular if enhancing nodules or "positive cytology" has been verified [144, 145]. Targeted biopsy of the cyst wall and solid components has a significant incremental diagnostic value over cyst-fluid aspiration alone for the diagnosis of mucinous and malignant cystic neoplasms [383, 401, 402] and is recommended by the ESGE guideline on EUS-guided sampling in gastroenterology [54]. EUS and CE-EUS significantly improve the discrimination and characterization of mural nodules in IPMN in comparison with B-mode EUS and CT [403-406], suggesting that CE-EUS may facilitate EUS-guided sampling of solid components in PCL.

\section{Subepithelial gastrointestinal tumours}

Differential diagnosis of hypoechoic gastric SETs by EUS and other imaging techniques is difficult $[66,178,179,191,407]$. Preliminary findings suggest that CE-EUS can discriminate GIST (hypervascular) from benign lesions (leiomyoma, lipoma: hypovascular) [408].

\section{Recommendation 34}

Contrast-enhanced EUS and/or EUS-elastography may be used to guide and target EUS-guided sampling of lymph nodes (LoE 2a), solid pancreatic masses (LoE 2a), pancreatic cystic lesions (LoE 2b) and gastrointestinal subepithelial tumours (LoE 3b) (GoR C). Strong consensus (100\%).

\section{Recommendation 35}

In addition to aspiration of cyst fluid, the cyst wall and solid components, if present, should be sampled in pancreatic cystic lesions (LoE 2b, GoR B). Strong consensus (100\%).

\section{EUS-guided sampling: Processing and evaluation of specimen \\ $\nabla$}

Techniques for expelling a specimen from the needle The techniques for expelling a specimen are related to the needle system used. There are three techniques for expelling a specimen from aspiration needles: 1. Air flushing; 2. Saline flushing; 3. Extrusion with the stylet. No differences between air flushing and stylet reinsertion with regard to the number of diagnostic specimens, overall accuracy and specimen quality were found in an RCT, but smears were bloodier following stylet use for the expelling of the sample [365].

\section{Recommendation 36}

Material can be expelled from EUS-aspiration needles using the stylet, by spraying with air on slides, or by flushing the needle with saline (LoE 2b, GoR B). Strong consensus (100\%).

\section{Recommendation 37}

Controlled release of material onto slides using the stylet is the preferred method despite more blood contamination (LoE 5, GoR D). Strong consensus (100\%).

\section{Methods for specimen preparation and processing}

Optimizing specimen handling and processing may reduce the frequency of non-diagnostic samples [409, 410]. The handling of the specimen depends on the diagnostic methods used and the final cytopathological evaluation.

\section{Cytopathology: conventional smears and liquid-based preparations}

For cytological analysis the specimen should be smeared onto slides (smear cytology, SC) or incorporated into special preservation solutions (liquid-based cytology, LBC) for further handling [411, 412]. Prospective comparative trials demonstrated that LBC was less sensitive with material obtained by EUS-guided sampling from lymph nodes and pancreatic lesions compared with traditional SC [413-415]. A further RCT suggested that LBC may serve as a complementary preparation technique if blood contamination of smears is abundant [414].

\section{Histopathology: cell block and core tissue preparation} In general, histopathological processing including paraffin-embedding, microtome-sectioning, and staining is possible with small coherent tissue cores embedded in a formalin fixative or with CB. A wide variety of ancillary diagnostic techniques (immunohistochemistry, molecular analysis) may be performed on histopathologically processed material $[66,186,382,409,411,412$, 416-420].

CB may be prepared from any cell suspension (e.g. liquid aspirates, needle rinses, or specimen in preservative solutions for LB). Pellets of artificially aggregated cells are obtained by using repeated centrifugation and/or special cell capturing gels [410, 412, 417, 421].

Small core particles obtained with aspiration needles or "histology needles" can be directly delivered into formalin solution for fixation followed by standard histopathological processing [53, 217, 409, 410, 422, 423]. Alternatively, core particles may be isolated from samples expelled on glass slides or preservation solutions by the stylet method before further cytopathological processing ("split-up" method) [186, 382, 424].

\section{Cytopathological evaluation of a specimen}

\section{Cytopathological vs. histopathological evaluation}

In most cases of PDAC and lymph node metastases, cytopathological evaluation alone is adequate to establish a diagnosis of malignancy. The diagnostic sensitivity of EUS-FNA cytology is highly dependent on the proficiency and experience of the cytopathologist evaluating the specimen $[107,425]$ and to a lesser extent on the availability of ROSE.

In a number of clinical settings and target lesions, it is tissue architecture, immunohistochemistry and molecular analysis rather 
than cellular features that are essential for accurate pathological assessment [419, 420]. For benign diseases, e.g. autoimmune pancreatitis [426, 427], differential diagnosis of SET (GIST vs. leiomyoma or schwannoma) [52, 181, 189, 191, 428, 429], subtyping of NSCLC and malignant lymphoma [217, 266, 430 -432], diagnosis of rare tumours [137, 226, 382], grading of neuroendocrine tumours [433 -435], molecular profiling of solid tumours [124$128,130,204-209,409,436,437]$ and differential diagnosis of mediastinal lymph node metastases [52, 226, 438], a core sample is preferred to a cytological aspirate [66].

\section{A complementary approach: combining cytopathological and histopathological assessment}

There are two reasonable strategies to achieve high diagnostic accuracy in EUS-guided sampling, without the attendance of a cytopathologist: combining cytopathological and histopathological evaluation [52, 138, 192, 231, 382, 415, 424, 439, 440], or relying on core tissue or CB material for histopathological assessment [53, 55, 217, 333, 409, 422, 423, 427, 430, 441 -444]. Several studies have shown an incremental yield of combining SC with CB technique or histological core assessment in terms of diagnostic yield and accuracy [138, 192, 231, 382, 415, 439, 440, 445, 446].

\section{Specimen handling for diagnosis of infectious diseases}

Suspected infectious disease (e.g. abscesses, tuberculosis or atypical mycobacteriosis) requires specific handling of the aspirate. Microbiological cultures are possible if samples are placed in saline, whereas a polymerase chain reaction (PCR) amplifying mycobacterial DNA requires the specimen placed in formalin [447, 448]. A moderate to high accuracy of SC including Ziehl-Neelsen stain for acid-fast bacteria alone for the diagnosis of tuberculosis and its differentiation from sarcoidosis or histoplasmosis has been reported [447, 449-454]. Further studies showed significantly higher sensitivity of PCR vs. microbiological culture and/ or SC, and of microbiological culture vs. SC [448, $455-457]$. In a meta-analysis the overall diagnostic yield of EBUS-TBNA for the diagnosis of mediastinal tuberculous lymphadenitis was $80 \%$ with the culture positive rate (54\%) being significantly higher than the SC positive rate (33\%) [458].

\section{Recommendation 38}

The processing of specimen obtained by EUS-guided sampling varies according to the cytopathological or histopathological methods used for diagnosis (LoE 2b, GoR C). Strong consensus $(100 \%)$.

\section{Recommendation 39}

Tissue fragments can be effectively isolated from the whole sample after gross visual examination without impairing the cytopathological result (LoE $2 \mathrm{~b}, \mathrm{GoR}$ B). Strong consensus (100\%).

\section{Recommendation 40}

Without rapid on-site cytopathological evaluation, the combination of cytopathological and histopathological processing seems to provide the most reliable results (LoE $2 b$, GoR B). Strong consensus (100\%).
Recommendation 41

Specimens obtained with EUS-guided sampling can be used for special ancillary studies including microbiological culture, biochemical analysis, immunocytochemistry, immunohistochemistry, and molecular analysis (LoE 2b, GoR B). Strong consensus $(100 \%)$.

\section{Recommendation 42}

Liquid-based techniques may be used complementary to traditional smearing for cytopathological evaluation of specimens (LoE 2b, GoR C). Broad agreement (90\%).

\section{Recommendation 43}

For the diagnosis of tuberculosis, smear cytology including Ziehl-Neelsen stains should be combined with microbiological culture of specimens transferred into sterile saline, and PCR amplification of mycobacterial DNA using formalin-fixed specimens (LoE 2b, GoR B). Strong consensus (100\%).

On-site assessment of the adequacy of specimens Rapid on-site cytopathological evaluation

ROSE is reported to be used in the majority of centres in the United States [106, 329], but on-site cytopathological service is not routinely available in Europe [327, 328]. Positive assessments of the role of ROSE for EUS-FNA of solid pancreatic lesions and for EUS-FNA and EBUS-TBNA of lymph nodes have been documented $[138,375,413,459-471]$. A high concordance of preliminary onsite interpretation and final cytopathological diagnoses has been reported $[460,466,472,473]$. Other studies have suggested that ROSE is not essential for high diagnostic yield and accuracy of EUS-FNA [54, 66, 474-476]. Only five studies with EUS-FNA of pancreatic lesions used a 2-cohort design with head-to-head comparison of adequacy and accuracy of ROSE vs. non-ROSE. These studies have been included in a meta-analysis, showing that ROSE was associated with a significant $10 \%$ improvement of the per-case adequacy rate only in studies with a low adequacy rate $(<90 \%)$. No significant impact of ROSE on diagnostic yield, accuracy or number of needle passes was observed [477]. In a second meta-analysis of 68 single-cohort and 2 two-cohort studies, ROSE was associated with a low, statistically significant advantage of adequacy rates (2.3\%). [478]. A further meta-analysis showed a non-significantly higher per-case adequacy in ROSE studies compared with studies without ROSE [136]. The largest meta-analysis assessing the diagnostic performance of EUS-FNA of solid pancreatic lesions demonstrated significantly lower heterogeneity among studies with a cytopathologist present on-site, but advantages over studies without ROSE in terms of sensitivity ( 88 vs. $80 \%$ ) and diagnostic odds ratio did not reach statistical significance [132].

Two RCTs showed no benefit of ROSE in EBUS-TBNA for diagnosing lung cancer and in EUS-FNA of solid pancreatic lesions with regard to diagnostic accuracy, procedure time and safety. However, in both trials ROSE-assisted sampling required fewer needle passes to obtain a diagnosis [467, 467a].

The relatively small benefit of ROSE has to be balanced against the cost and time expenditure of a permanent on-site cytopathology service [377, 479-481]. 
Gross visual inspection and ROSE performed by the endosonographer

In the absence of ROSE, gross visual assessment of a sample to assess the adequacy of EUS-guided sampling is possible [482]. Studies show conflicting levels of the ability of endosonographers and trained EUS and cytology assistants to assess slide adequacy and to differentiate malignant and benign specimens [483]. In a prospective evaluation of the visual assessment of core particles obtained with a $19 \mathrm{G}$ ProCore needle, a core length of $\geq 4 \mathrm{~mm}$ was an indicator for an adequate histological sample and for a high overall, histological and cytological diagnostic yield [443].

Studies have demonstrated the improved ability of endosonographers, following completion of structured training, to smear and stain slides, to operate a microscope [484], to judge adequacy of a cytological specimen confidently [485], and to differentiate benign and malignant smears with an accuracy of $89 \%$ [486]. ROSE performed by an experienced endosonographer may be reliable, but requires completion of an intensive structured training program.

\section{Recommendation 44}

Rapid on-site cytopathological assessment does not substantially improve the diagnostic yield of malignancy or generally reduce the number of needle passes. In centres with an adequacy rate $<90 \%$, ROSE may improve the adequacy of EUSguided sampling (LoE 2a, GoR C). Strong consensus (100\%).

\section{Recommendation 45}

Rapid on-site cytopathological assessment may be used to guide the number of needle passes, provide a reliable immediate diagnosis of malignancy, and assess the need for ancillary studies (LoE 2b, GoR C). Strong consensus (100\%).

\section{Reporting and reliability of cytopathology in EUS-guided sampling} Cytopathological reporting

In most cytopathological laboratories, the diagnostic categories for EUS-cytology follow a Bethesda-like system: unsatisfactory, benign, atypical, suspicious, and positive for malignancy ${ }^{11}[487,488]$. Based on a standard nomenclature for FNA [489], the Papanicolaou Society of Cytopathology has developed guidelines on standardized terminology and nomenclature for pancreatobiliary cytopathology, recommending a six-tiered system of diagnostic categories: nondiagnostic, negative, atypical, neoplastic, suspicious, and positive ${ }^{12}$. The category "neoplastic" is unique for pancreatic cytopathology, including benign neoplasms (serous cystadenoma) as well as premalignant mucinous cysts, neuroendocrine tumours and solid pseudopapillary neoplasms [490]. The inconclusive and ill-defined diagnostic categories "atypical" and "suspicious for malignancy"13 are inconsistently conveyed [491-493]. Insufficient adherence to standardized nomenclature in cytopathological reporting hampers individual patient management and is a substantial obstacle for the comparison of results between centres and studies [412, 490].

\footnotetext{
${ }^{11}$ Terms are explained in the addendum on terminology (long version only).

12 Terms are explained in the addendum on terminology (long version only).

${ }^{13}$ Terms are explained in the addendum on terminology (long version only).
}

Reliability of negative ("benign") results and findings positive for malignancy/ neoplasia

A few studies have analysed the reproducibility of cytopathological diagnoses of specimens obtained by EUS-guided sampling. In most studies a good-to-excellent diagnostic agreement between experienced cytopathologists was documented [425, 494, 495].

For clinical decision-making it is important to understand that there is still a significant risk of malignancy with negative findings in EUS-guided sampling, which is considerably high for PCL, solid pancreatic lesions in patients with underlying chronic pancreatitis, indeterminate biliary strictures, and gastrointestinal SET. The major cause for false-negative diagnoses of EUS-guided sampling is sampling error, whereas interpretative error is rare [66, 165, 325, 349, 350, 491, 492, 496 - 501].

In studies with good reference standards and long follow-up, false-positive findings have been reported for EUS-guided sampling in $1.1-5.3 \%$ when only cases with a positive cytopathological result were considered and in $7.8 \%$ if suspicious cytopathology results were included. False-positive rates seem to be higher in luminal compared with extra-luminal (e.g. pancreatic) primary cancer $[238,502,503]$. False-positive findings may result from procedure-related factors, e. g. traversal of a neoplastic area, needle contamination with tumour cells [240, 241], inadvertent aspiration of cancer-cell contaminated luminal fluid [239], or from cytopathological misinterpretation [66, 325, 491, 496 - 498, 501, $502,504,505]$.

\section{Malignancy risk associated with inconclusive findings}

Studies have evaluated the malignancy risk related with inconclusive diagnostic cytopathological categories for EUS-guided sampling of pancreatic lesions. The suspicious category is associated with an $80-96 \%$ risk of neoplastic or malignant final diagnosis, whereas the atypical category carries a markedly variable and significantly lower malignancy risk [492, 493, 506]. Clinical predictors associated with a high likelihood of a neoplastic diagnosis following an atypical cytopathological diagnosis in pancreatic EUS-FNA can be used to adapt management of patients with inconclusive cytopathological diagnoses: ancillary testing, watchful waiting, repeat EUS-guided sampling, alternative diagnostic techniques, or initiation of a specific treatment [501, 507-510]. Repeat EUSguided sampling in patients with high clinical suspicion of malignancy has been shown to yield a conclusive and correct diagnosis in $73-84 \%$ of cases with primarily inconclusive cytopathological diagnosis [468, 507, 510-512].

\section{Recommendation 46}

A standardized and validated classification system should be used for cytopathology reporting in EUS-guided sampling (LoE 5, GoR D). Strong consensus (100\%).

\section{Recommendation 47}

After inconclusive cytopathological results, the use of ancillary studies and/or repeat EUS-guided sampling should be weighed against the clinical background of patient history, clinical and laboratory data, EUS morphology, results of other imaging tests and follow-up (LoE 2b, GoR B). Strong consensus (100\%). 


\section{Adverse events of EUS-guided interventions and their prevention \\ $\nabla$}

\section{General considerations}

EUS-guided sampling (including EBUS-TBNA) is a safe diagnostic technique. Severe adverse events are rare. There is no evidence that needle size or needle type affects morbidity of EUS-guided sampling [54, 70, 330, 513]. A systematic review of complications of 51 EUS-FNA studies including 10941 patients describes an overall complication rate of $0.98 \%$. The procedure-related mortality was estimated at $0.02 \%$ [514].

Morbidity of EUS-guided therapeutic interventions is considerably higher than EUS-guided sampling, with risk and type of complications depending on the type of procedure $[71,515,516]$.

\section{Adverse events}

Perforation

The majority of perforations associated with EUS-guided sampling are caused by the passage of the gastrointestinal echoendoscope and not the sampling procedure. Multicentre surveys and studies report perforation rates of $0.03-0.15 \%$ [516-520]. The risk of duodenal perforation is higher than oesophageal perforation $[70,516]$.

\section{Acute pancreatitis}

The frequency of acute pancreatitis following EUS-FNA of pancreatic lesions is between $0.19-2.35 \%$ [70]. Pancreatitis risk is higher in patients with PCL than in patients with solid pancreatic lesions $[70,514]$.

\section{Intra- and extraluminal haemorrhage}

Severe bleeding following EUS-FNA is a rare event (overall $0.13 \%$ ) [514]. There are three reports in the literature of fatal bleeding following pancreatic EUS-FNA [70]. There is indirect evidence that EUS-FNA of PCL may cause a higher risk of bleeding compared with EUS-FNA of solid pancreatic lesions [514, 515, 521 - 523]. Guidelines suggest discontinuing oral anticoagulants, heparin and LMWHs in therapeutic dosages as well as ADP-antagonists before EUS-guided sampling, whereas withdrawal of ASA is recommended only before EUS-FNA of PCL [54, 513, 524 - 526]. Despite limited evidence for distinct cut-off values, a platelet count $<50000 / \mathrm{ml}$ and an international normalized ratio (INR) $>1.5$ are regarded as contraindications to EUS-guided sampling. There is no data regarding needle size, needle type, number of needle passes or other technical factors related to bleeding risk following EUS-guided sampling.

\section{Recommendation 48}

In patients on antiplatelets and/or anticoagulants, a risk assessment balancing thromboembolic events versus bleeding should be performed prior to EUS-/EBUS-guided diagnostic and therapeutic interventions (LoE 5, GoR D). Strong consensus $(100 \%)$.

\section{Recommendation 49}

Decision on suspension of antiplatelet drugs and/or anticoagulants or delay of the procedure should be made based on an individual risk assessment (LoE 5, GoR D). Strong consensus $(100 \%)$.

\section{Fever and infection}

Bacteremia following upper as well as lower gastrointestinal tract EUS-FNA is a rare event [527-530]. In large series 0.4$1.0 \%$ of patients experienced febrile episodes following EUS-FNA for solid pancreatic masses [359, 531, 532]. For endosonographic sampling of mediastinal lesions, studies report an overall frequency of major infectious complications in $0.07 \%$ of patients [533]. Serious infectious complications have been reported following EUS-FNA of mediastinal cysts [70, 533], ascites [313], perirectal cysts [26], as well as of PCL and (peri-) pancreatic fluid collections [534]. Despite a lack of convincing evidence, several guidelines recommend peri-interventional antibiotic treatment for EUS-FNA of PCL $[54,513,524]$. Due to potentially catastrophic consequences of mediastinal infection, guidelines and reviews regard EUS-FNA of mediastinal cysts to be contraindicated [54, $70,513,524,533]$.

\section{Recommendation 50}

Transoesophageal EUS-guided sampling of cystic mediastinal lesions should be avoided due to the high risk of infection (LoE 4, GoR C). Strong consensus (100\%).

\section{Recommendation 51}

Peri-interventional antibiotic treatment is recommended for EUS-guided sampling of cystic lesions and fluid collections as well as in EUS-guided drainage procedures (LOE 5, GOR D). Strong consensus (100\%).

\section{Tumour cell seeding and needle track metastasis}

Several studies agree that EUS-guided sampling for malignant solid and cystic pancreatic tumours and for cholangiocarcinoma is not a risk factor for the development of peritoneal seeding, tumour recurrence, or decreased survival [535-541]. The risk of peritoneal seeding following biopsy of pancreatic cancer appears to be significantly lower with EUS-FNA compared to percutaneous FNA [542, 543]. However, 11 individual cases of tumour cell seeding caused by EUS-guided sampling of malignant pancreatic neoplasia ( $n=8$, in 4 cases cystic tumours) [544-551] and of lymph node metastases $(n=3)$ [552 - 554] suggest cautious use of EUS-guided sampling.

\section{Recommendation 52}

Tumour cell dissemination along the needle track following EUS-guided sampling is an exceptionally rare event. Pre-operative EUS-guided sampling of pancreatobiliary malignancies is not associated with increased risk of postoperative recurrence, decreased overall survival or decreased cancer-specific survival (LoE 2c, GoR B). Strong consensus (100\%).

\section{Miscellaneous complications}

There are reports of bile peritonitis following EUS-FNA of the gallbladder or of the liver in patients with biliary obstruction [293, 555, 556], of pneumothorax following transoesophageal EUS-FNA of mediastinal lymph nodes [202], pneumoperitoneum following EUS-FNA of a pancreatic tumour [557] and of pancreatic duct leak caused by EUS-FNA of pancreatic mass lesions [558, 559]. One case of phlegmonous gastritis following EUS-FNA has 
been reported [560]. Two cases of a hypertensive crisis induced by EUS-FNA of retroperitoneal paraganglioma or adrenal phaeochromocytoma have been documented [561, 562].

\section{Risk factors for complications}

There are limited data linking complication rates of EUS-FNA with specific target lesions. Data from a systematic review confirmed that EUS-FNA is exceptionally safe for mediastinal lesions ( $n=1310$; complication rate: $0.38 \%)$, abdominal masses $(n=381$; $0.26 \%$ ) and adrenal glands $(n=81 ; 0 \%)$. Procedure-related morbidity seems to be higher for pancreatic lesions ( $n=8246$ : $1.03 \%$ ), liver lesions ( $n=344 ; 2.33 \%)$ and perirectal lesions $(n=193: 2.07 \%)$ [514]. Several studies have shown that performing EUS with/without EUSguided sampling and ERCP in a single session is safe [563 - 570]. Observations from high-volume centres suggest that complications are more frequent in the learning phase of the examiners $[100,422,534]$.

\section{Recommendation 53}

EUS-guided sampling of solid lesions is a low-risk procedure. EUS-guided sampling of pancreatic cystic lesions has a higher frequency of adverse events compared to solid pancreatic lesions (LoE 2a, GoR B). Strong consensus (100\%).

\section{Recommendation 54}

EUS-guided sampling and ERCP may be safely performed in a single session (LoE 2b, GoR B). Strong consensus (100\%).

\section{Recommendation 55}

Any complications of EUS-guided sampling and therapeutic interventions should be documented in a standardized format in every centre. The incidence of adverse events should be monitored and used as a quality indicator (LoE 5, GoR D). Strong consensus (100\%).

\section{Affiliations}

1 Department of Internal Medicine, Krankenhaus Märkisch Oderland Strausberg/Wriezen, Germany

2 Department of Internal Medicine 2, Helios Hospital Meiningen GmbH, Meiningen, Germany

3 Gastroenterology Unit, Department of Medical and Surgical Sciences University of Bologna/Hospital of Imola, Italy

${ }^{4}$ National Centre for Ultrasound in Gastroenterology, Haukeland University Hospital, Bergen and Department of Clinical Medicine, University of Bergen, Bergen, Norway

${ }^{5}$ Departement of Gastroenterology and Endoscopy, Ospedale Maggiore Crema, Italy

${ }^{6}$ Department of Medicine and National Centre for Ultrasound in Gastroenterology, Haukeland University Hospital, Bergen, Norway

7 Department of Internal Medicine 2, Caritas Krankenhaus, Bad Mergentheim, Germany

8 Department of Gastroenterology, Research Center of Gastroenterology and Hepatology, University of Medicine and Pharmacy, Craiova, Romania and Department of Endoscopy, Gastrointestinal Unit, Copenhagen University Hospital Herlev, Denmark

${ }^{9}$ GastroUnit, Department of Surgery, University Hospital Herlev, Copenhagen, Denmark

10 Department of Internal Medicine I, Sana Kliniken, Luebeck, Germany

11 Department of Gastric Surgery, Ultrasound Section, Herlev Hospital, University of Copenhagen, Denmark

12 Department of Gastroenterology, Brandenburg University of Medicine Theodor Fontane, Neuruppin, Germany

13 Department of Radiology, GB Rossi University Hospital, University of Verona, Italy

14 Unit of Internal Medicine, Department of Medical and Surgical Sciences, University of Bologna, Italy

${ }^{15}$ Department of Radiology, King's College Hospital, London, UK

16 Department of Internal Medicine 2, Caritas Krankenhaus, Bad Mergentheim, Germany

17 Sino-German Research Center of Ultrasound in Medicine, The First Affiliated Hospital of Zhengzhou University, Zhengzhou, China

\section{Acknowledgement}

\section{$\nabla$}

We acknowledge the advice from Lynne Rudd, EFSUMB general secretary, and the support of the EFSUMB INVUS committee.

\section{References \\ (References online)}

